\title{
Forced Magnetic Reconnection and Field Penetration of an Externally Applied Rotating Helical Magnetic Field in the TEXTOR Tokamak
}

\author{
Y. Kikuchi, ${ }^{1}$ M. F. M. de Bock, ${ }^{2}$ K. H. Finken, ${ }^{1}$ M. Jakubowski, ${ }^{1}$ R. Jaspers, ${ }^{2}$ H. R. Koslowski, ${ }^{1}$ A. Kraemer-Flecken, ${ }^{1}$ \\ M. Lehnen, ${ }^{1}$ Y. Liang, ${ }^{1}$ G. Matsunaga,${ }^{3}$ D. Reiser, ${ }^{1}$ R. C. Wolf,${ }^{1}$ O. Zimmermann, ${ }^{1}$ and TEXTOR-team \\ ${ }^{1}$ Institut fuer Plasmaphysik, Forschungszentrum Juelich GmbH, EURATOM Association, D-52425, Germany \\ ${ }^{2}$ FOM Institute for Plasma Physics Rijnhuizen, EURATOM Association, Postbus 1207, NL-3430 BE Nieuwegein, The Netherlands \\ ${ }^{3}$ Fusion Research and Development Directorate, Japan Atomic Energy Agency, 311-0193, Japan
}

(Received 4 June 2006; published 23 August 2006)

\begin{abstract}
The magnetic field penetration process into a magnetized plasma is of basic interest both for plasma physics and astrophysics. In this context special measurements on the field penetration and field amplification are performed by a Hall probe on the dynamic ergodic divertor (DED) on the TEXTOR tokamak and the data are interpreted by a two-fluid plasma model. It is observed that the growth of the forced magnetic reconnection by the rotating DED field is accompanied by a change of the plasma fluid rotation. The differential rotation frequency between the DED field and the plasma plays an important role in the process of the excitation of tearing modes. The momentum input from the rotating DED field to the plasma is interpreted by both a ponderomotive force at the rational surface and a radial electric field modified by an edge ergodization.
\end{abstract}

DOI: 10.1103/PhysRevLett.97.085003

PACS numbers: $52.30 . \mathrm{Cv}, 52.35 . \mathrm{Vd}$

The penetration of a magnetic field into a magnetized plasma is a fundamental interest for the plasma physics community, but the topic is not easily accessible. Besides the classical skin effect, a forced magnetic reconnection [1] at the resonance surface plays an important role; the latter process is faster than the classical skin effect and is connected with the excitation of tearing modes. It has been observed that the excitation of the tearing modes requires a threshold of the perturbation field. The excitation of the tearing mode is preceded by a braking of the toroidal plasma rotation $[2,3]$. This behavior of so-called "mode penetration" is understood as a result of bifurcation of the forced magnetic reconnection [4].

The TEXTOR tokamak is equipped with the dynamic ergodic divertor (DED) which consists of 16 external perturbation coils at the high field side inside the vacuum vessel [5]. The DED system can produce "static" or "rotating" helical magnetic perturbation fields up to a maximum frequency of $10 \mathrm{kHz}$ which covers the frequency range of the typical plasma diamagnetic drift of the TEXTOR tokamak. The base mode numbers of the DED field can be selected as $m / n=12 / 4,6 / 2$, and $3 / 1$ by changing the coil connections. The DED in $m / n=3 / 1$ configuration produces a strong sideband component of $m / n=2 / 1$, so that a $m / n=2 / 1$ tearing mode phase locked by the DED field is well reproduced [6]. In this Letter, we focus on a study of field penetration of the rotating helical magnetic perturbation field. It is shown that a shielding current is induced at the resonant surface which leads to a reduction of the field penetration, a force transfer to the plasma, and a change of the phase of the edge magnetic perturbation field. The observed mode penetration process will be discussed with a theoretical analysis based on a linearized four-field model.
Previous work on the tearing mode had shown that the excitation of a tearing mode in the plasma depends strongly on the plasma rotation; a rotation in plasma current direction destabilizes the mode in a certain range of rotation frequencies, whereas counterrotation has a stabilizing influence [6]. The threshold exhibits a minimum when the frequency of the external perturbation $f_{\mathrm{DED}}$ equals the MHD frequency of the mode $f^{\prime}=f_{\text {tor }}-f_{\text {DED }}-f_{*, e} \simeq 0$ at $q=2$, where $f_{\text {tor }}$ is the toroidal plasma rotation frequency and $f_{*, e}$ is the electron diamagnetic frequency [7]. The tearing mode structure has been measured by a soft $\mathrm{x}$ ray camera that gave an island width of typically $6 \mathrm{~cm}$ to $8 \mathrm{~cm}$ depending on plasma conditions [8].

The present analysis is extended by measurements of a Hall probe which measures the magnetic field created by both the shielding of the external perturbation field at the resonant surface and after the excitation of the tearing mode the electrical current in the island. The Hall probe is installed on a movable probe holder at the low field side equatorial midplane; it is absolutely calibrated and measures $B_{r}$ and $B_{\theta}$ components of the magnetic field; the frequency characteristics cover the frequency range of the dynamic operation of the DED [9]. In this study the Hall probe was located at $r=49 \mathrm{~cm}(r / a=1.04)$. The toroidal plasma rotation at the resonant surface is measured by charge exchange recombination spectroscopy (CXRS). The TEXTOR tokamak is equipped with two neutral beam injection (NBI) systems, which are oriented in the opposite toroidal directions. Dependencies of the threshold of the DED coil current for the mode penetration on the toroidal plasma rotation have been investigated in terms of the above NBI systems in TEXTOR [7].

As compared to Mirnov coils, the Hall probe has the advantage that it can also be operated in dc field without 
integration and be moved closer to the plasma. However, for these measurements we analyzed discharges with a rotating external field of $1 \mathrm{kHz}$ in order to guarantee the detection of the full mode amplitude by a single probe. The external frequency of the DED field is considered as the main plasma response frequency. The time development of the plasma response to the DED field is shown in Fig. 1. The input power levels of the two NBI systems are balanced in the discharge, so that the toroidal rotation is almost zero at $q=2$ unless the DED is applied. The plasma parameters were a plasma current, $I_{p}$ of $300 \mathrm{kA}$, a toroidal magnetic field, $B_{t}$ of $2.25 \mathrm{~T}$, a line-averaged electron density, $\bar{n}_{e}$ of $2.0 \times 10^{19} \mathrm{~m}^{-3}$, a major radius, $R_{0}$ of $1.75 \mathrm{~m}$, and a minor radius, $a$ of $0.47 \mathrm{~m}$. The toroidal projection of the rotation direction of the DED field was set to be opposite to the plasma current, and the driving frequency of the DED was $1 \mathrm{kHz}$. Here, the rotation direction of the DED corresponds to the electron diamagnetic drift when projecting it to the poloidal direction. After the plasma parameters reached stationary conditions, the DED coil current was ramped up slowly to clarify nonlinear behaviors of the field penetration.

Figure 1(a) displays the amplitude of the magnetic field component at $f=1 \mathrm{kHz}$ (DED frequency); a dashed line, the value of the vacuum field, is given for comparison. Figures 1(b) and 1(c) show the phase relative to the external field and the plasma rotation. Two stages in the time development of the plasma response to the DED field can be distinguished as follows. First, no change of the electron

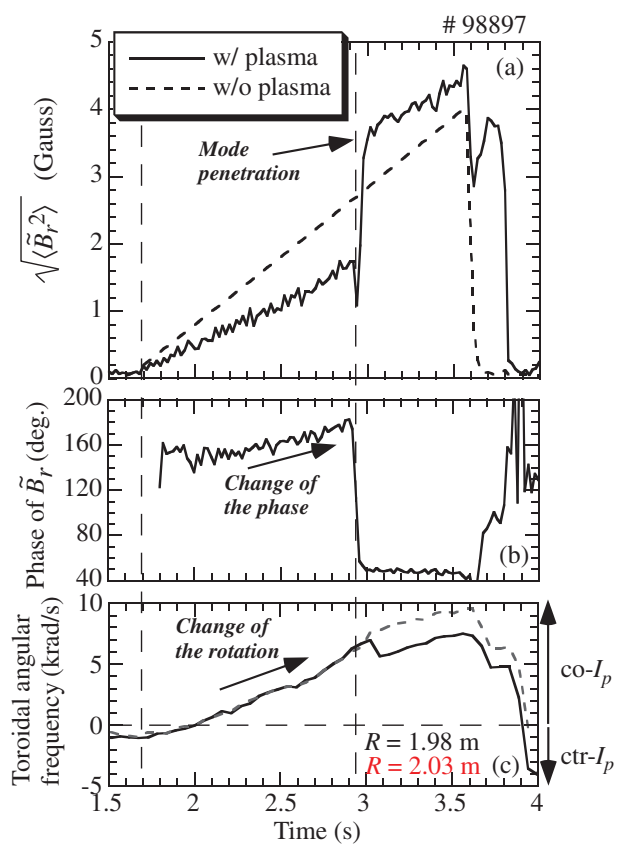

FIG. 1 (color online). Time evolutions of (a) the amplitude of radial magnetic perturbation fields with (solid line) and without (dashed line) the plasma, (b) the phase with respect to the DED coil current waveform, (c) the toroidal angular velocities at $R=$ $1.98 \mathrm{~m}$ (solid line), and $R=2.03 \mathrm{~m}$ (dashed line). temperature and the density is observed before the DED coil current reaches the threshold for the mode penetration. Even though the DED rotates in the counterdirection with respect to $I_{p}$, the background toroidal plasma rotation velocity increases in the codirection as shown in Fig. 1(c). It is noticed that the phase of the plasma response to the DED starts to change as well as the time development of the toroidal plasma rotation during this period. The second stage is characterized by the excitation of the $m / n=2 / 1$ tearing mode. The onset of the tearing mode is identified at $t=2.9 \mathrm{~s}$ by sudden changes of the amplitude and the phase of the magnetic perturbation field, where the DED coil current is about $1.9 \mathrm{kA}$. The experimental data of Fig. 1(a) show clearly that in the first phase the induced current is phased such that it shields the penetrating perturbation field; after passing the threshold a phase jump is observed and the current at the resonant surface amplifies the external perturbation.

Even though there are some sideband components of the DED field, it can be assumed that the sudden increase of the amplitude of the magnetic perturbation field originates from the contribution of the $m / n=2 / 1$ tearing mode. From the observed magnetic perturbation field, the width of the magnetic island of $m / n=2 / 1$ is estimated to be $\sim 6 \mathrm{~cm}$ in terms of the following well-known formula of $w=4\left(r_{s} q \tilde{B}_{r}\left(r_{s}\right) / m B_{\theta 0} q^{\prime}\right)^{1 / 2}[10]$, where $w, r_{s}, \tilde{B}_{r}, B_{\theta 0}, q^{\prime}$ are the island width, the radial position of $q=2$, the radial component of the magnetic perturbation field, the equilibrium poloidal magnetic field, and the radial derivative of the $q$, respectively; this island width is in good agreement with the evaluation by the soft $\mathrm{x}$-ray camera. If it is assumed that the tearing mode current is not shielded by electrical currents between the resonance layer and the probe, the electrical current in the tearing mode can be derived to $\delta I_{\text {mode }} \simeq 1.8 \mathrm{kA}$.

A model has been developed to explain the penetration process, specifically of the DED field. We have performed to reproduce the mode onset by the DED field in terms of a quasilinear MHD simulation code [11]. However, effects of plasma diamagnetic drift are neglected in the singlefluid MHD approximations. Therefore, we have extended the model to a linearized four-field model [12] in cylindrical coordinates. The magnetic field is given by $\boldsymbol{B}=B_{0} e_{z}+$ $\boldsymbol{\nabla} \times\left(A_{\|} e_{z}\right)$, where $B_{0}$ is the toroidal magnetic field and $A_{\|}$ is the vector potential in the parallel direction (the direction of $z$ axis). Here we assume a constant electron temperature and a cold ion limit. In addition, effects of electron inertia and toroidal curvature are neglected for simplicity. The linearized four-field model is described as follows:

$$
\begin{gathered}
\frac{\partial \tilde{n}}{\partial t}=\frac{1}{B_{0}} \frac{i m}{r}\left(\tilde{\phi} \frac{d n_{0}}{d r}+\frac{1}{e} \tilde{A}_{\|} \frac{d j_{\| 0}}{d r}\right)+\frac{1}{e} i k_{\|} \tilde{j}_{\|} \\
-i k_{\|} n_{0} \tilde{\boldsymbol{v}}_{\|}+D \nabla_{\perp}^{2} \tilde{n}, \\
\frac{\partial \tilde{A}_{\|}}{\partial t}=-i k_{\|} \tilde{\phi}+i k_{\|} \frac{k_{B} T_{e}}{n_{0} e} \tilde{n}-\frac{i m}{r} \tilde{A}_{\|} v_{\mathrm{de}}+\frac{\eta}{\mu_{0}} \nabla_{\perp}^{2} \tilde{A}_{\|},
\end{gathered}
$$




$$
\begin{gathered}
\frac{\partial \tilde{U}}{\partial t}=\frac{B_{0}^{2}}{\rho} i k_{\|} \tilde{j}_{\|}+\frac{B_{0}}{\rho} \frac{i m}{r} \tilde{A}_{\|} \frac{d j_{\| 0}}{d r}+\frac{\mu_{\perp}}{\rho} \nabla_{\perp}^{2} \tilde{U} \\
\frac{\partial \tilde{v}_{\|}}{\partial t}=-\frac{k_{B} T_{e}}{\rho B_{0}} \frac{i m}{r} \tilde{A}_{\|} \frac{d n_{0}}{d r}-\frac{1}{\rho} i k_{\|} k_{B} T_{e} \tilde{n}+\frac{\mu_{\|}}{\rho} \nabla_{\perp}^{2} \tilde{v}_{\|},
\end{gathered}
$$

where $k_{\|}=\left(m B_{\theta 0} / r B_{0}-n / R_{0}\right)$ is the parallel component of the wave vector of the perturbations, $v_{\mathrm{de}}=$ $-\left(k_{B} T_{e} / e B_{0} n_{0}\right)\left(d n_{0} / d r\right)$ is the electron diamagnetic drift velocity, and $j_{\| 0}=(\nu+1) I_{p} /\left(\pi a^{2}\right)\left\{1-(r / a)^{2}\right\}^{\nu}$ is the equilibrium plasma current density. Here, $\tilde{\phi}$ is the perturbed plasma potential, $\tilde{v}_{\|}$is the perturbed parallel velocity, $\tilde{n}$ is the perturbed plasma density, $\tilde{U}\left(=\nabla_{\perp}^{2} \tilde{\phi}\right)$ is the perturbed vorticity, $B_{\theta 0}$ is the equilibrium poloidal field, $R_{0}$ is the major radius, $I_{p}$ is the plasma current, and $\nu$ is the peaking factor of the plasma current density profile. The superscript $\sim$ and the subscript 0 denote the perturbed fields and the equilibrium quantities, respectively. The perturbations are assumed to be described by a single Fourier mode of $m / n=2 / 1$ in the poloidal and the toroidal directions, so that effects of mode coupling with higher harmonics are not taken into account in the present analysis. In addition to the plasma resistivity, the density diffusivity $D$, the perpendicular ion viscosity $\mu_{\perp}$, and the parallel ion viscosity $\mu_{\|}\left(=4 \mu_{\perp}\right)$ are introduced as the additional dissipation terms. The diffusion coefficients are set in this study as $D=1.0 \mathrm{~m}^{2} / \mathrm{s}$ and $\mu_{\perp}=\mu_{\perp 0}$. Here, the $\mu_{\perp 0}$ is the neoclassical perpendicular viscosity $(=7.8 \times$ $10^{-10} \mathrm{~kg} \mathrm{~m}^{-1} \mathrm{~s}^{-1}$ ). The DED coil current is given in the vacuum region outside the plasma in the computation. The numerical treatment has been described in [13].

Dependencies of the absolute value and the phase of $\tilde{A}_{\|}$ on the DED frequency at $q=2$ are shown in Fig. 2. One strong resonance is identified at $f_{\mathrm{DED}}=f_{*, e}$, where the $f_{\mathrm{DED}}$ and the $f_{*, e}$ are the DED frequency and the electron diamagnetic drift frequency, respectively. A similar shift of the resonance to $f_{*, e}$ has also been described in a kinetic model on the penetration of an error field [14]. Figure 3 shows the radial profiles of the perturbed current density induced by the DED. A shielding current at around $q=2$ can be identified in the case of $+1 \mathrm{kHz}$. This case corresponds to the condition just after the application of the DED in the real experiment as shown in Fig. 1, where the toroidal plasma rotation is almost zero at around $q=2$, and the magnetic perturbation field is attenuated in comparison with the vacuum field. On the other hand, the radial structure of the $\tilde{j}_{\|}$is changed when the DED frequency is set at around $f_{*, e}$ as shown in Fig. 3(b). It is considered that the strong amplification of the $\tilde{A}_{\|}$in Fig. 2(a) is originated from the modification of the $\tilde{j}_{\|}$. It is noticed again that the toroidal plasma rotation is not included in the present model, so that the resonant condition of the field penetration is satisfied by changing the driving frequency of the DED. The perpendicular force, $F_{\perp}$, induced by the DED is evaluated by the formula of $F_{\perp}=2 \pi R_{0} \int_{0}^{a}\left\langle\tilde{j}_{\|} \tilde{B}_{r}\right\rangle 2 \pi r d r$

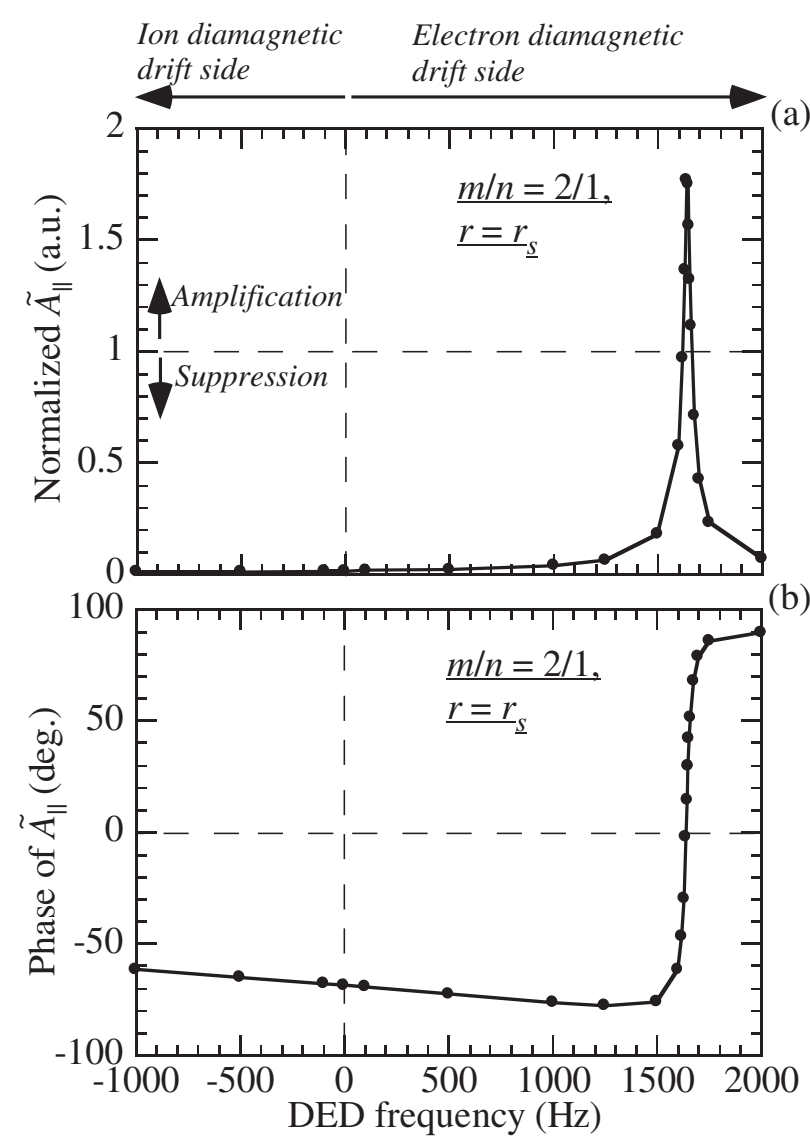

FIG. 2. Frequency dependencies of (a) the magnitude and (b) the phase of the $\tilde{A}_{\|}$at $r=r_{s}$, where $T_{e}=1 \mathrm{keV}, n_{0}=$ $2.5 \times 10^{19}\left[1-(r / a)^{2}\right]+5 \times 10^{18} \mathrm{~m}^{-3}, \quad I_{p}=300 \mathrm{kA}, \quad B_{0}=$ $2.25 \mathrm{~T}, \nu=8, m / n=2 / 1, \tilde{B}_{(m, n)=(2,1)}=6.7 \mathrm{G}$ at $q=2$. The diffusivities are set as $D=1.0 \mathrm{~m}^{2} / \mathrm{s}$ and $\mu_{\perp}=\mu_{\perp 0}$.

(ponderomotive force). Dependencies of $F_{\perp}$ on the DED frequency are shown in Fig. 4. Remarkably, the force can transfer in the opposite direction of the rotation direction of the DED at a specific frequency range $\left(0<f_{\mathrm{DED}}<f_{*, e}\right)$. It
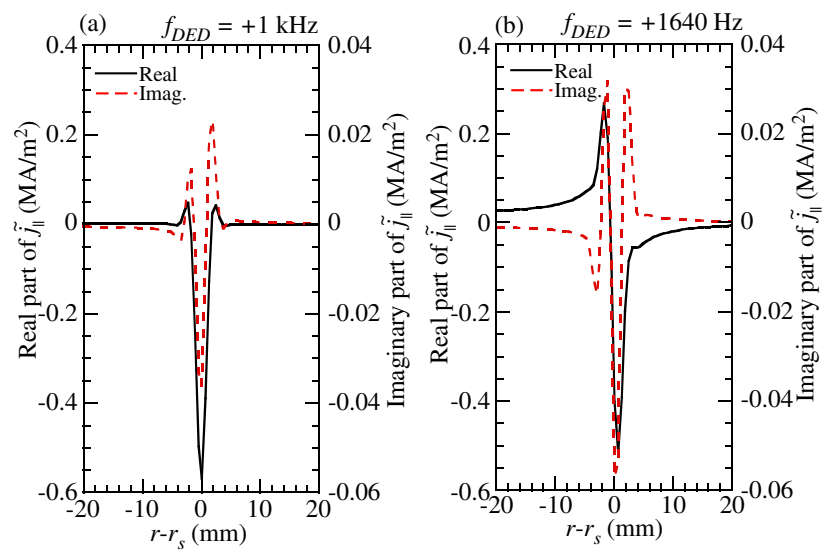

FIG. 3 (color online). Radial profiles of the perturbed parallel current, where the plasma parameters are the same as Fig. 2. (a) $f_{\mathrm{DED}}=+1 \mathrm{kHz}$, (b) $f_{\mathrm{DED}}=+1640 \mathrm{~Hz}\left(\sim f_{*, e}\right)$. Here, $D=$ $1.0 \mathrm{~m}^{2} / \mathrm{s}$ and $\mu_{\perp}=\mu_{\perp 0}$. 


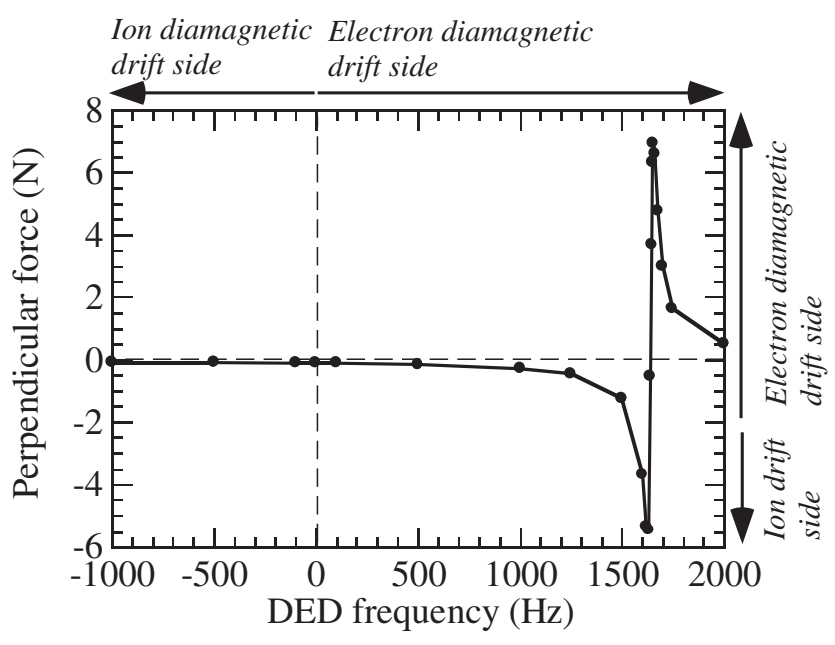

FIG. 4. Frequency dependencies of the perpendicular force by the DED, where the plasma parameters are the same as Fig. 2.

should be noticed that if the values of the diffusivities are very small values, an additional peak in the frequency dependence of the $F_{\perp}$ appears at $f_{\mathrm{DED}}=0$. The additional peak is originated from an effect of $\mathbf{E} \times \mathbf{B}$ drift as shown in a dispersion relation of drift-tearing mode [15].

The four-field model describes the initial phase of the field penetration depicted in Fig. 1. There, the experiment shows the shielding current which is obviously induced at the resonant surface(s) by the external field. This current produces a force on the plasma such that the plasma is accelerated or decelerated; if the condition $f^{\prime}=0$ is met, the tearing mode grows resonantly. In the initial phase, the toroidal plasma rotation gradually changes to the codirection (i.e., opposite to the rotation direction of the DED) before the mode penetration in the experiment. The shielding current prevents the generation of an island which would be imposed by the external field in the vacuum approximation (i.e., without the shielding current). For this reason the plasma temperature and density at the $q=$ 2 surface remains initially unchanged, i.e., $f_{*, e}$ at around $q=2$ is not modified by the DED before the mode penetration; only the toroidal plasma rotation is changed by the DED so that the $f^{\prime}$ approaches zero at $q=2$. Thus, the $f_{*, e}$ plays a role of the offset frequency for the resonant condition. This is the reason why the toroidal plasma rotation has a stabilizing effect, and the minimum value of the required DED coil current for the mode penetration is shifted from the condition where the toroidal plasma rotation at around $q=2$ is zero. The error bar of the plasma rotation for a single measurement may be too large for a definite statement but experiments changing either the plasma rotation or the DED frequency showed clearly the same effect [7]. On the other hand, there is a different physical picture to explain the change of the toroidal plasma rotation by the DED as shown in [16], which is originated from a change of radial electric field due to an edge ergodization [17]. The direction of the induced force by the ergodization is always the codirection in TEXTOR, so that it could be considered that an approach to the frequency matching is prevented by the effect of the ergodization in the case of $f^{\prime}>0$, whereas that is assisted in the case of $f^{\prime}<0$. This understanding also supports the experimental observations of the threshold for the mode penetration [7].

In summary, new measurements by a Hall probe have been performed on the field penetration into a tokamak plasma and the data have been investigated theoretically by means of a linearized four-field model. The model explains well the observed dynamic response of the tokamak plasma to the DED. The observed edge magnetic perturbation field suggests that the plasma response to the DED field relates to the change of the toroidal plasma rotation. The result of the role of the plasma flow versus the tearing mode velocity may be important for the general quantification of the field penetration process in magnetically confined plasmas.

One of the authors, Y. K., thanks to the financial support of Japan Society for the Promotion of Science (JSPS). Others thank the Deutsche Forschungsgemeinschaft for support by the SFB 591.

[1] T. S. Hahm and R. M. Kulsrud, Phys. Fluids 28, 2412 (1985).

[2] T. C. Hender et al., Nucl. Fusion 32, 2091 (1992).

[3] R. J. Buttery et al., Nucl. Fusion 39, 1827 (1999).

[4] R. Fitzpatrick, Phys. Plasmas 5, 3325 (1998).

[5] Special Issue on Dynamic Ergodic Divertor, edited by K. H. Finken [Fusion Eng. Des. 37, 335 (1997)].

[6] R. C. Wolf et al., Nucl. Fusion 45, 1700 (2005).

[7] H. R. Koslowski et al., Nucl. Fusion 46, L1 (2006).

[8] Y. Liang et al., Proceedings of the 32nd EPS Conference on Plasma Physics (ECA, Tarragona, 2005), Vol. 29C, p. 4.060.

[9] I. Duran et al., Rev. Sci. Instrum. 73, 3482 (2002).

[10] J. Wesson, Tokamaks (Clarendon, Oxford, 1997), 2nd ed.

[11] Y. Kikuchi et al., Plasma Phys. Controlled Fusion 48, 169 (2006).

[12] K. Biskamp, Magnetic Reconnection in Plasmas (Cambridge University Press, Cambridge, England, 2000).

[13] Y. Kikuchi et al., Contrib. Plasma Phys. (to be published).

[14] M. F. Heyn et al., Nucl. Fusion 46, S159 (2006).

[15] R. Fitzpatrick and T. C. Hender, Phys. Fluids B 3, 644 (1991).

[16] K. H. Finken et al., Phys. Rev. Lett. 94, 015003 (2005).

[17] I. Kaganovich and V. Rozhansky, Phys. Plasmas 5, 3901 (1998). 